\title{
A DESCRIPTIVE STUDY- CUTANEOUS MANIFESTATIONS OF DIABETES
}

\author{
Pooja Nupur1, Sweety Thakur², Yogesh Kumar Yashaswi ${ }^{3}$ \\ ${ }^{1}$ Senior Resident, Department of Skin and V. D, Nalanda Medical College and Hospital, Patna. \\ ${ }^{2}$ Skin Specialist, Sub-Divisional Hospital, Danapur, Patna. \\ ${ }^{3}$ Senior Resident, Department of Surgery, IGIMS, Sheikhpura, Patna.
}

\begin{tabular}{l}
\hline ABSTRACT \\
BACKGROUND \\
Diabetes is the most common non-communicable disease globally. Awareness of mucocutaneous manifestations may lead to \\
recognition of yet undiagnosed DM.
\end{tabular}

\section{MATERIALS AND METHODS}

It is a descriptive and observational study conducted on 75 diabetic patients. Investigations performed included urine for sugar, protein, fasting and post prandial blood sugar, blood urea, serum creatinine, lipid profile, liver function test and ECG. Special investigations like culture and sensitivity of pus, skin scrapings for fungal infections and biopsy of skin lesion was done in selected cases. Analysis was carried out using SPSS 10.

\section{RESULTS}

Of 75 patients examined, 60 patients (80\%) suffered from cutaneous lesions. Some patients suffered from more than one lesion; $50 \%$ of total cases suffered from infections. Diabetic dermopathy in our study was observed in $20 \%$ of the patients. Some of the cutaneous markers of diabetes mellitus like necrobiosis lipoidica diabeticorum, diabetic bullae, acquired perforating dermatosis and stiff skin syndrome is not seen in our study.

\section{CONCLUSION}

This study was done to highlight the importance of association of cutaneous lesions and diabetes mellitus.

\section{KEYWORDS}

Diabetes Mellitus, Cutaneous Manifestations, Diabetic Dermopathy.

HOW TO CITE THIS ARTICLE: Nupur P, Thakur S, Yashaswi YK. A descriptive study- cutaneous manifestations of diabetes. J. Evolution Med. Dent. Sci. 2017;6(71):5019-5022, DOI: 10.14260/Jemds/2017/1091

\section{BACKGROUND}

Diabetes is the most common non-communicable disease globally. It is estimated that nearly 380 million adults worldwide will have diabetes by 2025 . Diabetes among adults has reached approximately $20 \%$ in urban populations and $10 \%$ in rural population. It may be due to genetic defect, autoimmunity, defect in the formation of insulin or decreased insulin sensitivity. It may occur at any age, but greatest number of individuals with Diabetes was aged $45-64$ yrs. $^{1}$ DM increases with ageing. Prevalence is slightly greater in men than female. Risk factors are changes in lifestyle like diet, lack of dietary fibres, sedentary lifestyle, alcohol and stress. ${ }^{2}$ The cutaneous signs are the manifestations of multiple factors. Abnormal carbohydrate metabolism, other altered metabolic pathways, atherosclerosis, microangiopathy, neuronal degeneration and impaired host mechanism all play an important role. ${ }^{3}$ Cutaneous involvement has been found in $43 \%-66 \%$ of diabetic patients approximately. ${ }^{3}$ This study was done to highlight the importance of association of cutaneous lesions and diabetes mellitus.

Financial or Other, Competing Interest: None.

Submission 28-07-2017, Peer Review 21-08-2017,

Acceptance 28-08-2017, Published 04-09-2017.

Corresponding Author:

Dr. Pooja Nupur,

Senior Resident,

Department of Skin \& V. D,

Nalanda Medical College and Hospital,

Agam Kuan, Patna-800007.

E-mail: Pooja.nupur.it@gmail.com

DOI: $10.14260 /$ jemds $/ 2017 / 1091$

\section{(c) $(1)(5)$}

Awareness of mucocutaneous manifestations may lead to recognition of yet-undiagnosed DM. Better understanding of the burden of skin disorders in diabetic patients may raise awareness of prevention and management. Therefore, aim of study is to perform an observational study to evaluate the main clinical characteristics and complications of skin disorders in diabetic patients.

\section{MATERIALS AND METHODS}

The study was conducted in the Department of Dermatology and Venereology of Nalanda Medical College and Hospital over a period of 12 months from October 2015 - October 2016. All the diabetic patients attending the skin OPD and diabetic in patients with skin problems were examined over 12 months; 75 diabetic patients visiting the Department of Dermatology were examined for cutaneous findings. Out of 75 patients, 60 patients were found to have some cutaneous manifestations of diabetes mellitus; 15 out of 75 patients came for some psychocutaneous features, infestation (pediculosis) or without any obvious clinical findings and two never gave consent for the study. There were 38 males and 22 females, whose age ranged from 12 to 74 years of age; 55 patients were diagnosed with diabetes type-II and 5 patients were pre-diagnosed with type-I with an average duration of 7 years and 8 years respectively. Investigations performed included urine for sugar, protein, fasting and post prandial blood sugar, blood urea, serum creatinine, lipid profile, liver function test and ECG. Special investigations like culture and sensitivity of pus, skin scrapings for fungal infections and biopsy of skin lesion was done in selected cases wherever necessary. 


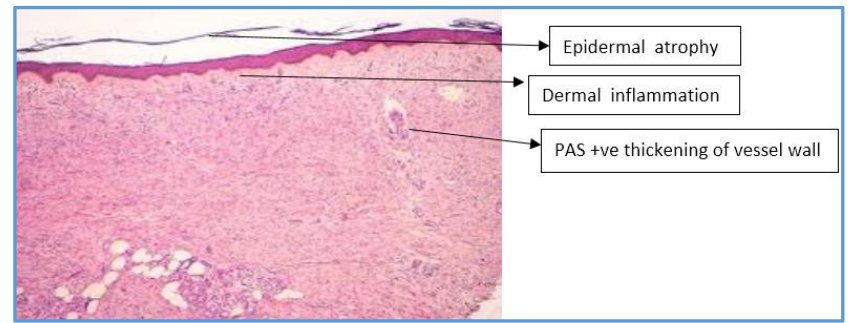

Figure 1. Shows Epidermal Atrophy, Mild Dermal Inflammation and often Microangiopathy with PAS +ve Thickening of Vessels Wall on Biopsy of Skin Spot

\section{RESULTS}

Of 75 patients examined, 60 patients (80\%) suffered from cutaneous lesions. Some patients suffered from more than one lesion. Out of 60 patients, $50 \%$ of total cases suffered from infections. Infection was the most common finding amongst patients; 15 patients had fungal infection, most commonly found was tinea as against other studies in which staphylococcus infection was found to be the most common. ${ }^{4}$

10 patients had furuncles, 4 had abscess and 1 patient suffered from cellulitis.

$20 \%(12)$ of diabetic patients had diabetic dermopathy as against $50 \%-70 \%$ in other studies. ${ }^{5}$ Out of 12 patients, 10 patients had bilateral involvement of feet and 2 patients had similar finding on forearm also.

$10 \%$ (6) of patients had foot involvement. Paraesthesia and pruritus was present in all the 6 patients; 4 patients had trophic changes with 2 patients suffering from foot ulcer.

Other common findings are skin tags (8.3\%), xanthelasma palpebrarum (3.34\%), acanthosis nigricans (6.67\%) and cherry angioma $(1.67 \%)$.

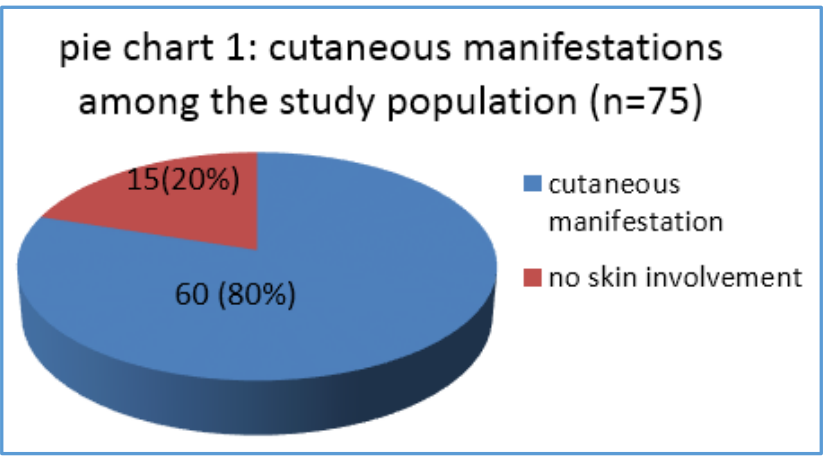

\begin{tabular}{|c|c|c|}
\hline $\begin{array}{c}\text { Cutaneous } \\
\text { Manifestations }\end{array}$ & Number & (\%) \\
\hline Infections & 30 & $50 \%$ \\
\hline $\begin{array}{c}\text { Diabetic } \\
\text { dermopathy }\end{array}$ & 12 & $20 \%$ \\
\hline Foot changes & 6 & $10 \%$ \\
\hline Skin tags & 5 & $8.32 \%$ \\
\hline $\begin{array}{c}\text { Acanthosis } \\
\text { nigricans }\end{array}$ & 4 & $6.67 \%$ \\
\hline $\begin{array}{c}\text { Xanthelasma } \\
\text { palpebrarum }\end{array}$ & 2 & $3.34 \%$ \\
\hline Cherry angioma & 1 & $1.67 \%$ \\
\hline Table 1. Cutaneous Manifestations of Diabetes (n= 60) \\
\hline
\end{tabular}

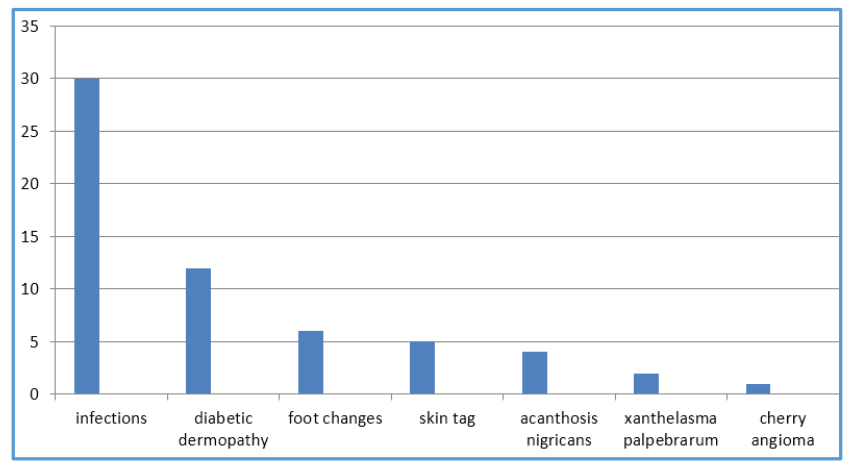

Table 2. Graphical representation of the Cutaneous Manifestations of Diabetes among 60 Patients

\section{DISCUSSION}

Cutaneous signs of diabetes mellitus are extremely valuable to the clinician. They generally appear after the primary lesion has developed, but may signal or appear coincidentally with its onset or even precede diabetes by many years.

Cutaneous manifestations of diabetes are classified into four categories: Skin lesions with strong-to-weak association to diabetes (necrobiosis lipoidica, diabetic dermopathy, diabetic bullae, yellow skin, eruptive xanthomas, perforating disorders, acanthosis nigricans, oral leucoplakia, lichen planus), infections (bacterial, fungal), cutaneous manifestations of diabetic complications (microangiopathy, macroangiopathy, neuropathy) and skin reactions to diabetic treatment (Sulphonylureas or insulin).4 Most of the dermatological manifestations are related to or a sequelae of diabetes mellitus. Pathogenesis includes impaired phagocytosis, delayed neutrophil chemotaxis, reduced leucocyte adherence and abnormal microcirculation may be contributory factors, especially during hyperglycaemia and diabetic acidosis. Cutaneous T-cell function also decreased in diabetes. It has been suggested that a large number of dermatological problems in diabetes are caused by hyperglycaemia accelerated production of non-enzymatic advanced glycosylated end products. ${ }^{1}$

Although prior studies showed increased risk of infection in DM patients, ${ }^{6}$ little evidence is found in the literature to support increased risk for cutaneous infectious diseases. ${ }^{7}$ In general, skin disorders are highly associated to poorly controlled DM patients. A good glycaemic control may reduce the incidence and severity of cutaneous disorders with or without known pathogenesis. Non-specific skin disorders that occur in diabetic patients can increase the likelihood of exposure to infectious organisms and contact with allergens, resulting in chronic and recurrent infections and eczemas respectively. However, further studies are required, since all datas are still not concordant.

Skin disorders in DM patients are highly correlated with glycaemic control. As an example, Foos et al conducted a study with 403 DM patients in Brazil and evaluated their skin disorders and glycaemic control. Thus, the study demonstrated $94 \%$ of patients with inadequate glycaemia control had some skin disorders. ${ }^{6}$

DM affects the skin through several mechanisms being hyperglycaemia per se and AGEs the most well described. Reaching pathological high levels of glycaemia strongly affects skin homeostasis by inhibiting keratinocyte proliferation and migration, protein biosynthesis, inducing endothelial cell apoptosis, decreasing nitric oxide synthesis 
and impairing phagocytosis and chemotaxis from several cells. Besides hyperglycaemia induce direct damage, high glucose levels also induce AGE formation. AGEs are formed from glycation of proteins, lipids and nucleic acids that act in several pathways including reactive oxygen species (ROS) formation, impairing ROS clearance as well as intra and extracellular proteins functions and inducing proinflammatory cytokine through nuclear factor $\mathrm{kB}(\mathrm{NF}-\mathrm{kB})$ pathway. ${ }^{6}$

Indeed, AGE biochemical interactions are one of the major pathways involved in DM complications including skin disorders. ${ }^{6}$ AGE alters collagen properties, decreasing flexibility and solubility and increasing its rigidity. Also, AGE participate in the development of fibrosis in DM, in skin aging and even in diabetes-related immunosuppression. Diabetes related immunosuppression affects the skin wounding, mainly by leucocyte impaired function and malfunction of growth factors.

In addition to the previously mentioned pathways, high glucose levels also impair the normal functioning of keratinocytes in vitro, decreasing its proliferation and differentiation. Moreover, keratinocytes studies are more commonly conducted in animal models with scarce data on diabetic human skin. ${ }^{2}$

Regarding epidermal thickness alterations, Bertheim et al demonstrated that diabetic patients with severe joint mobility on the hands had an increased epidermal thickness with abnormal hyaluronan distribution on skin layers, while Zakharov et al demonstrated that well-controlled patients with type $1 \mathrm{DM}$ did not present alteration on the epidermal thickness. This data reinforces that skin disorders in skin disorders in DM patients are strongly related to glycaemic control.

Perez and Kohn stated that about $30 \%$ of diabetics have some cutaneous involvement during the course of the disease. ${ }^{2}$ In our study, $80 \%$ of patients had some cutaneous findings. This shows higher incidence of skin involvement; $50 \%$ patients in our study presented with infections, most common cutaneous findings amongst them. In our study, fungal infection was the most common as compared to other studies in which bacterial infection is the commonest. ${ }^{5}$ This difference may be attributed to the poor socioeconomic patients with poor hygiene, humid and hot environmental factors, self-administration of wrong drug and negligent patients. Moreover, diabetic state decreases the resistance of host to infection by impairing the functions of neutrophils. ${ }^{4}$

Diabetic dermopathy is found in $30 \%-60 \%$ of patients and is the most common finding in diabetics. ${ }^{2}$ Diabetic dermopathy is a skin condition characterised by light brown or reddish, oval or round, slightly indented scaly patches most often appearing on shins. Diabetic dermopathy is sometimes referred to as shin spots or pigmented pretibial patches. In our study, this was observed in $20 \%$ of the patients. The exact pathogenesis is obscure, but it may be a cutaneous marker of microangiopathy at other sites. ${ }^{1}$ Diabetic dermopathy tends to occur in older patients or in those who have had diabetes for at least $10-20$ years. $^{2}$ It appears to be closely linked to increased glycosylated haemoglobin, an indicator of poor control of blood glucose levels. Patients presenting with shin spots not already diagnosed of diabetes should undergo further investigation to rule out the possibility of early diabetes. With good glycaemic control, they tend to disappear. ${ }^{2}$

$8.3 \%$ of patients in our study had foot involvement. All the 6 patients suffered from paraesthesia and pruritus; 4 patients were having trophic changes and 2 patients are having foot ulcer in our study. Due to the peripheral nerve dysfunction associated with diabetes (diabetic neuropathy), patients have reduced ability to feel pain. This means that minor injuries remain undiscovered for a long while. Research estimates that the lifetime incidence of foot ulcers within the diabetic community is around $15 \% .^{2}$ Precipitating factors for foot ulcer are- abnormal plantar pressure points, foot deformities, ill-fitting shoes and socks, callus formation, poor glycaemic control, long duration of diabetes and previous foot ulceration. In diabetes, peripheral nerve dysfunction can be combined with peripheral artery disease (PAD) causing poor blood circulation to the extremities (diabetic angiopathy). Around half of patients with a diabetic foot ulcer have co-existing PAD. ${ }^{4}$

Other common findings are skin tags $(8.3 \%)$ in our study. Skin tags are small, pedunculated, soft often pigmented lesions occurring on the eyelids, the neck and the axillae. A few studies have reported an association between multiple skin tags and diabetes and between skin tags and insulin resistance. Crook $^{7}$ found that skin tags were associated with the typical atherogenic lipid profile seen in the insulin resistant states: elevated triglycerides and low levels of high density lipoprotein. Skin tags can be regarded as a sign of impaired glucose tolerance, diabetes and increased cardiovascular risk. ${ }^{7}$

In our study, Xanthelasma palpebrarum was found in two patients (3.34\%). Xanthelasma palpebrarum (XP) are yellow plaques that occur most commonly near the inner canthus of eyelid and are often associated with atherosclerosis, dyslipidaemia and coronary artery disease. Xanthelasma have a tendency to progress, coalesce and become permanent. Xanthelasma represent areas of macrophage containing lipids, primarily cholesteryl esters, but the exact pathogenesis is not known. Xanthelasma is composed of xanthoma cells, which are foamy histiocytes laden with intracellular fat deposits primarily within the upper reticular dermis. Most studies have found increased concentrations of plasma total cholesterol or low-density lipoprotein in people with xanthelasma. It is known to be associated with atherosclerosis, coronary artery disease, insulin resistance, diabetes mellitus, hypertension, stroke, dyslipidaemia, obesity and hyperuricaemia. ${ }^{8}$ Eruptive xanthomas are skin manifestation of a severe hypertriglyceridaemia and implicate an elevated risk for acute pancreatitis or type 2 Diabetes Mellitus. In our study, we never came across cases of eruptive xanthomas. To fully understand the pathogenesis of xanthelasma in Diabetes Mellitus and its association with it, further studies needs to be done in this regard.

In our study, 4 cases of acanthosis nigricans (6.67\%) in diabetic patients were encountered. Acanthosis nigricans most likely caused by factors that stimulate the epidermal keratinocyte and dermal fibroblast proliferation. In benign form of acanthosis nigricans, the factor is probably insulin or insulin like growth factor (IGF) that incites the epidermal cell proliferations. Other proposed mediators include other tyrosine kinase receptors (epidermal growth factor receptor (EGFR) or fibroblast growth factor receptors (FGFR). At high 
concentrations, insulin may exert potent proliferative effects via high affinity binding to IGF-1 receptors. In addition, free IGF-1 levels may be elevated in obese patients with hyperinsulinaemia, leading to accelerated cell growth and differentiation. Familial and syndromic forms of acanthosis nigricans have been identified. Many syndromes share common features including obesity, hyperinsulinaemia and craniosynostosis. In $1976 \mathrm{Kahn}$ et al published their landmark study, in which the association between acanthosis nigricans and insulin resistance was first described. In 2000, the American Diabetes Association established acanthosis nigricans as a formal risk factor for the development of diabetes in children. ${ }^{9}$

In our study, 1 patient had cherry angioma (1.67\%). Cherry angiomas are common skin growths that can develop on most of the areas of body. They are also known as the senile angiomas of body or Campbell de Morgan spots. They are usually found in people of 30 years and older. The broken blood vessels inside the cherry angioma give them a reddish appearance. The exact cause of cherry angiomas is not known, but there may be a genetic factor that makes certain people more likely to get them. They have also been linked to pregnancy, exposure to chemicals and climate. ${ }^{10}$ According to study conducted by Anterpreet Arora et al, amongst diabetics, cherry angiomas were observed in $44.8 \%$ of cases. Amongst the non-diabetics, cherry angiomas were recorded in $40 \%$ cases. Increased number has been reported in diabetics, but this result was not found to be significant. Further study needs to be done in this regard to find out whether association of cherry angioma in diabetic patient is significant or not.

Some of the cutaneous markers of diabetes mellitus like necrobiosis lipoidica diabeticorum (NLD is a degenerative disease of connective tissue seen in almost $50 \%$ of diabetic patients. Its strong association with diabetes has led to its name necrobiosis lipoidica diabeticorum), diabetic bullae, acquired perforating dermatosis (APD), granuloma annulare, oral lichen planus, vitiligo and stiff skin syndrome is not seen in our study. Therefore, we suggest further studies to be done in this regard.

\section{CONCLUSION}

We emphasise that our study demonstrates the high incidence of dermatological diseases in diabetic patients. The most common skin lesion found in our study is dermatophytoses followed by diabetic dermopathy. Some of the cutaneous markers of diabetes mellitus like necrobiosis lipoidica diabeticorum, diabetic bullae, acquired perforating dermatosis and stiff skin syndrome is not seen in our study. Unlike what the literature suggests, our data showed only few cases of acanthosis nigricans as well as cherry angiomas. Further study needs to be done in this regard to establish the relationship of acanthosis nigricans and Campbell de Morgan spot. By detecting the finding of diabetic dermopathy and other systemic findings of Diabetes Mellitus, we support the recent concept of using shin spot or diabetic dermopathy as a marker of internal complications.

This study was done to highlight the importance of association of cutaneous lesions and diabetes mellitus. Awareness of mucocutaneous manifestations may lead to recognition of yet undiagnosed DM. Better understanding of the burden of skin disorders in diabetic patients may raise awareness of prevention and management.

\section{REFERENCES}

[1] Huntley AC. Cutaneous manifestations of diabetes mellitus. Dermatol Clin 1989;7(3):531-46.

[2] Perez MI, Kohn SR. Cutaneous manifestations of diabetes mellitus. J Am Acad Dermatol 1994;30(4):519-31.

[3] Sibbald RG, Schachter RK. The skin and diabetes mellitus. Int J Dermatol 1984;23(9):567-84.

[4] Rayfield EJ, Ault MJ, Keusch GT, et al. Infection and diabetes: the case for glucose control. Am J Med 1982;72(3):439-50.

[5] Lawley TJ, Yancey KB. Diabetes mellitus. In: Eisen F, Wolff, Freedberg, et al. eds. Dermatology in general medicine. New York: McGraw-Hill Book Company 1993:123-9.

[6] Foss NT, Polon DP, Takada MH, et al. Dermatoses em pacientes com diabetes mellitus. Revista de Saude Publica 2005;39(4):677-82.

[7] Crook MA. Skin tags and the atherogenic lipid profile. J Clin Pathol 2000;53(11):873-4.

[8] Gangopadadhya DN, Dey SK, Mitra C, et al. Serum lipid profile in xanthelasma. The Indian Journal of Dermatology 1998;43(2):53-7.

[9] Sinha S, Schwartz RA. Juvenile acanthosis nigricans. J Am Acad Dermatol 2007;57(3):502-8.

[10] Cohen AD, Cagnano E, Vardy DA. Cherry angiomas associated with exposure to bromides. Dermatology 2001;202(1):52-3. 